\title{
High concordance of programmed death-ligand 1 expression with immunohistochemistry detection between antibody clones 22C3 and E1L3N in non-small cell lung cancer biopsy samples
}

\author{
Wei Zhang", Ziyang Cao", Caixia Gao, Yan Huang, Chunyan Wu, Liping Zhang, Likun Hou \\ Department of Pathology, Shanghai Pulmonary Hospital, Tongji University School of Medicine, Shanghai, China \\ Contributions: (I) Conception and design: L Zhang, L Hou; (II) Administrative support: C Wu, Y H; (III) Provision of study materials or patients: \\ C Gao, Z Cao; (IV) Collection and assembly of data: W Zhang, Z Cao; (V) Data analysis and interpretation: W Zhang, L Hou; (VI) Manuscript \\ writing: All authors; (VII) Final approval of manuscript: All authors. \\ "These authors contributed equally to this work. \\ Correspondence to: Liping Zhang; Likun Hou. Department of Pathology, Shanghai Pulmonary Hospital, Tongji University School of Medicine, \\ Shanghai 200433, China. Email: Lipingzhang2002@outlook.com; 1600027@tongii.edu.cn.
}

Background: The detection of programmed death-ligand 1 (PD-L1) expression can enrich for patients who respond to anti-programmed cell death 1 (PD-1)/PD-L1 therapies. Though, for most laboratories, the cost of PD-L1 22C3 pharmDx is prohibitive for widespread use, whereas the laboratory-developed test (LDT) PD-L1 E1L3N antibody clone is widely available and inexpensive. This study aims to explore the analytical performance of E1L3N on the Dako Autostainer Link-48 platform and further evaluate the concordance of E1L3N and 22C3 expression in non-small cell lung cancer (NSCLC) biopsy samples.

Methods: A total of 171 NSCLC biopsy samples were utilized in this study. Cases with less than 100 tumor cells were excluded. Serial sections of representative blocks were used for immunohistochemistry (IHC) staining. The staining protocol was performed according to the standard PD-L1 IHC 22C3 pharmDx package. PD-L1 staining on the tumor cell membrane was detected by immunofluorescence.

Results: At a $1 \%$ cutoff value, PD-L1 was positive in $46.2 \%$ of patients using clone $22 \mathrm{C} 3$ and $42.1 \%$ of patients using E1L3N assays. At a 50\% cutoff value, PD-L1 was positive in $16.4 \%$ of patients using clone $22 \mathrm{C} 3$ and $15.2 \%$ of the patients using E1L3N assays. Cohen's kappa was used to evaluate the concordance of the PD-L1 expression between clone 22C3 and E1L3N. The kappa values were 0.893 [95\% confidence interval (CI): $0.826-1$ ] at the $1 \%$ cutoff and 0.868 (95\% CI: $0.764-1)$ at the $50 \%$ cutoff. An evaluation of the intraclass correlation coefficients (ICCs) between the antibodies was used to quantify the interassay variability for PD-L1 expression in tumor cells. ICCs showed high concordance between the two antibodies (0.955, 95\% CI: 0.939-0.967). Cohen's kappa was also used to assess the consistency of the PD-L1 evaluation between two pathologists. The kappa values were 0.941 and 0.912 at the $1 \%$ cutoff, and 0.904 and 0.909 at the $50 \%$ cutoff for clone $22 \mathrm{C} 3$ and E1L3N expression, respectively.

Conclusions: The results indicated that the clone E1L3N assay has a high concordance with 22C3. The PD-L1 clone E1L3N assay is reliable and cost-effective, and could be used as a primary screening agent for PD-L1 IHC staining in pathological laboratories, especially in a research setting.

Keywords: PD-L1 (22C3); PD-L1 (E1L3N); immunohistochemistry (IHC); non-small cell lung cancer (NSCLC); biopsy

Submitted Jan 02, 2020. Accepted for publication Jul 27, 2020.

doi: $10.21037 /$ tcr-20-101

View this article at: http://dx.doi.org/10.21037/tcr-20-101 


\section{Introduction}

Statistically, lung cancer has the highest incidence and mortality of all cancers among both sexes in the United States (1) and globally (2). Immunotherapy elucidates new treatment options for malignant tumors. Immune checkpoint inhibitors, such as anti-programmed cell death (PD-1) and anti-programmed death-ligand 1 (PD-L1), have proven to be highly effective in the treatment of different tumor types (3-5). The KEYNOTE-001 and KEYNOTE-024 trials demonstrated that the antiPD-1 inhibitor, pembrolizumab, significantly prolonged progression-free survival and overall survival for previously untreated non-small cell lung cancer (NSCLC) patients, with at least $50 \%$ of the tumor cells expressing PD-L1. Remarkably, pembrolizumab monotherapy can be extended as a first-line therapy to locally-advanced or metastatic NSCLC patients, with $1 \%$ (or more) of the tumor cells expression PD-L1 (6). PD-L1 22C3 PharmDx, on the dedicated Dako Autostainer platform, is an approved companion diagnostic assay for the detection of the PD-L1 immunotherapy biomarker (7), in addition to epidermal growth factor receptor (EGFR), anaplastic lymphoma kinase (ALK) and ROS proto-oncogene 1 (ROS1), which need to be tested in advanced NSCLC patients.

PD-L 1 protein expression is detected by immunohistochemistry (IHC). The PD-L1 Blueprint Projects 1 (8) and 2 (9) analyzed the concordance of PD-L1 clone expression across different detection platforms. Except for SP142, the PD-L1 clone 22C3, 28-8, and SP263 assays showed a similar expression pattern of tumor cells in NSCLC. Also, a real-world study that recruited 1,930 patients, including 412 lung cancer patients, reported that the 22C3 and 28-8 assays had analytical concordance (10). These studies indicate that 22C3, 28-8 and SP263 are interchangeable for the assessment of PD-L1 expression of tumor cells.

The E1L3N antibody clone has been detected in various malignant tumors including: melanoma (11), bladder (12), gastric (13), prostate (14), and breast (15). Previous studies have shown that E1L3N expression had a high concordance with the PD-L1 clones SP263 (16) and 28-8 (17). Several reports noted a close analytical performance between $22 \mathrm{C} 3$ and SP263 expression (18-21). However, different studies have reported conflicting results about evaluating the comparability of these PD-L1 clones assays. For example, clone 22C3 and SP263 assays (Dako and Ventana platform, respectively) exhibited a significant discrepancy in the positive percentage $(22,23)$. Ma et al. reported that E1L3N showed poor staining of gastric tumor cells compared to SP142 and 28-8 in surgical specimens on different detection platforms (13). Similarly, the E1L3N assay using Ventana BenchMark XT automated platform showed lower PDL1 positivity of bile duct tumor cells than SP263 and 22C3 assays in tissue microarrays (TMA) or whole tissue sections (24). The PD-L1 expression status in NSCLC biopsy samples using 22C3 and E1L3N antibodies on the Dako AutostainerLink-48 platform remains only partially understood. Moreover, standardized PD-L1 assays are costly, while PD-L1 clone E1L3N (for research use only) is inexpensive and widely available, and can be performed locally.

Therefore, this current study was undertaken to analyze and evaluate the analytical performance of the PD-L1 antibody clone E1L3N in comparison to the clone $22 \mathrm{C} 3$ on the Dako platform to assess its diagnostic value as a screening tool for NSCLC biopsy samples.

We present the following article in accordance with the STROBE reporting checklist (available at http://dx.doi. org/10.21037/tcr-20-101).

\section{Methods}

\section{Patients and reagents}

Our study was observational and retrospective and followed the World Medical Association's Declaration of Helsinki (as revised in 2013) and approved by the Ethics Committee of Shanghai Pulmonary Hospital (No. k17-130, Shanghai, China). Informed consent was taken from all the patients.

One hundred seventy-one primary NSCLC patients were included in the present study and were enrolled in the Shanghai Pulmonary Hospital between May 2018 and September 2018. Clinical information data was collected from the electronic medical record management system, including age, gender, smoking history, stage, histological subtypes and targeted gene mutations. Cases with numbers of tumor cells $(<100)$ were excluded. The PD-L1 antibody clone E1L3N was purchased from Cell Signaling Technology, and was derived from rabbit (Cat No. 13684S), and the immunogen was derived from intracellular peptides. The clone 22C3 was a Dako product (Cat No. M3653) that was derived from the extracellular peptides in mice (Cat No. S2022) (Figure S1). 


\section{Immunofluorescence staining}

Sections were deparaffinized and rehydrated, and antigen retrieval was performed. The immunofluorescence staining was conducted with Dako mouse anti-PD-L1 22C3 (1:50) paired with Yeasen anti-mouse IgG labeled with Alexa Fluor 594 (1:100) and Cell Signaling Technology rabbit antiPD-L1 E1L3N (1:400) paired with Yeasen anti-rabbit IgG labeled with Alexa Fluor 488 (1:100). All of the images were captured with ZEISS Imager.Z2 at a magnification of $\times 400$.

\section{Immunobistochemistry staining}

Serial sections of biopsy samples were prepared for IHC. PD-L1 staining using the two primary antibodies was performed on the Dako AutostainerLink-48 platform, according to the manufacturer's instructions. The concentrated antibody clone E1L3N was applied at a dilution of 1:400 using a visualization system (K8002, without LINKER). The concentrated antibody clone $22 \mathrm{C} 3$ was used at a dilution of 1:50 with a visualization system (K8002, with Mouse LINKER). The slides were counterstained with hematoxylin and mounted with cover slips.

\section{Evaluation of PD-L1 tumor positive percentage}

The assessment of PD-L1 expression was performed blindly by two senior thoracic pathologists who undertook a PDL1 testing (22C3) and evaluation training course (22C3) of NSCLC samples. The PD-L1 staining results were evaluated using the tumor proportion score (TPS), with the final score for each assay being defined as the mean of the pathologists' scores

The visualization of clear membranous positive staining in the tumor cells was classified as a positive result (excluding non-tumor cells and cytoplasmic staining of tumor cells) as described in the PD-L1IHC 22C3 pharmDx Interpretation Manual (25), irrespective of the staining intensities. Tonsil tissue was used as both a positive and negative control.

\section{Statistical analysis}

Scatterplots and Bland-Altman plots were used to show the concordance and differences between the two datasets. The plots were generated in Stata V14 and GraphPad Prism7. The overall percent agreement (OPA), positive percent agreement (PPA), negative percent agreement (NPA), and
Cohen's Kappa and Intra-Class Correlation (ICC) were calculated to determine the concordance of both datasets. All statistics were analyzed with SPSS v25.

\section{Results}

\section{Patient characteristics}

A total of 171 primary NSCLC patients who underwent biopsies were analyzed, including 94 squamous cell carcinomas (SQC) and 65 adenocarcinomas (ADC). The median age of the participants was 63 years old (range, 23-88 years old), with 135 (78.9\%, 135/171) males. The smoking rate was $38.6 \%(66 / 171)$. The proportion of early staged patients (stage I-II) was $3.5 \%$. Targeted therapyrelated driver genes were tested in 100 NSCLC cases (58.5\%, 100/171), including EGFR, Kirsten rat sarcoma oncogene (KRAS), and B-RAF proto-oncogene (BRAF) mutation, and ROS1 and ALK fusion genes. The results are shown in Table 1.

\section{TPS distribution of bothPD-L1 antibodies}

TPS evaluation was performed according to the Agilent $22 \mathrm{C} 3$ pharmDx manual (25) and the technical companion assay $(26,27)$. The three categories $(0,1-49 \%, \geq 50 \%)$ are illustrated with representative images of the staining pattern (scanned by MoticEasyScan), as shown in Figure 1. Immunofluorescence staining of NSCLC biopsy samples (Figure 2) with clones $22 \mathrm{C} 3$ and $\mathrm{E} 1 \mathrm{~L} 3 \mathrm{~N}$ revealed specific positive staining for $\mathrm{PD}-\mathrm{L} 1$ on the tumor cell membrane. E1L $3 \mathrm{~N}$ and 22C3 exhibited a strong correlation at the average PD-L1 expression (Figure 3A). The mean difference between the two assays in all cases was identified using Bland-Altman analysis (Figure 3B), and no significant differences were detected. E1L3N showed a high similarity with $22 \mathrm{C} 3$ at $1 \%$ and $50 \%$ cutoffs (Figure 3C).

\section{Reproducibility of the pathologists' evaluation scores}

For the clone $22 \mathrm{C} 3$ and E1L3N assays, the two pathologists' scores exhibited a high level of consistency (Figure $4 A, B, C, D, E, F)$, with no significant differences detected (Pitman's Test, $\mathrm{P}=0.120$ and $\mathrm{P}=0.428$, Figure 4). At the $1 \%$ and $50 \%$ cutoffs, the $22 \mathrm{C} 3$ expressions for Pathologist A were $47.4 \%$ and $46.8 \%$, respectively, and $15.8 \%$ and $15.8 \%$ for Pathologist B, respectively. Also, for the clone E1L3N assay, it was evaluated with positivity rates of 
Table 1 Clinical-pathological characteristics of patients

\begin{tabular}{|c|c|}
\hline Variables & Value \\
\hline Age (years), median [range] & 63 [23-88] \\
\hline \multicolumn{2}{|l|}{ Gender, n (\%) } \\
\hline Male & $135(78.9)$ \\
\hline Female & $36(21.1)$ \\
\hline \multicolumn{2}{|l|}{ Smoking, n (\%) } \\
\hline Yes \& ever & $66(38.6)$ \\
\hline No & $105(61.4)$ \\
\hline \multicolumn{2}{|l|}{ Histology, n (\%) } \\
\hline ADC & $65(38.0)$ \\
\hline SQC & $94(55.0)$ \\
\hline Favor LCNEC & $2(1.2)$ \\
\hline Favor PSC & $2(1.2)$ \\
\hline NSCLC & $8(4.6)$ \\
\hline \multicolumn{2}{|l|}{ T stage, $\mathrm{n}(\%)$} \\
\hline $\mathrm{T} 1$ & $8(4.7)$ \\
\hline $\mathrm{T} 2$ & $26(15.2)$ \\
\hline T3 & $28(16.4)$ \\
\hline $\mathrm{T} 4$ & $83(48.5)$ \\
\hline Unknown & 26 (15.2) \\
\hline \multicolumn{2}{|l|}{$\mathrm{N}$ stage, n (\%) } \\
\hline No & $12(7.0)$ \\
\hline $\mathrm{N} 1$ & $20(11.7)$ \\
\hline $\mathrm{N} 2$ & $60(35.1)$ \\
\hline N3 & $53(31.0)$ \\
\hline Unknown & $26(15.2)$ \\
\hline \multicolumn{2}{|l|}{ M stage, n (\%) } \\
\hline MO & $52(30.4)$ \\
\hline M1 & $80(46.8)$ \\
\hline Unknown & $39(22.8)$ \\
\hline \multicolumn{2}{|l|}{ TNM, n (\%) } \\
\hline I & $4(2.3)$ \\
\hline II & $2(1.2)$ \\
\hline III & $46(26.9)$ \\
\hline IV & $80(46.8)$ \\
\hline Unknown & $39(22.8)$ \\
\hline
\end{tabular}

Table 1 (continued)
Table 1 (continued)

\begin{tabular}{lc}
\hline Variables & Value \\
\hline Gene mutation, $\mathrm{n}(\%)$ & \\
ALK & $9(5.3)$ \\
BRAF & $1(0.6)$ \\
EGFR & $23(13.5)$ \\
KRAS & $4(2.3)$ \\
ROS1 & $3(1.8)$ \\
WT & $60(35.1)$ \\
Unknown & $71(41.5)$
\end{tabular}

PSC, pulmonary sarcomatoid carcinoma; LCNEC, large cell neuroendocrine carcinoma.

$41.5 \%$ and $43.9 \%$ for Pathologist $\mathrm{A}$ at the $1 \%$ and $50 \%$ cutoffs, respectively, and $15.8 \%$ and $14.6 \%$ for Pathologist $\mathrm{B}$, respectively (Table 2). Both pathologists achieved high overall reliability in evaluating the two PD-L1 assays.

\section{The concordance of both PD-L1 antibody clones and pathologists' evaluations}

The concordance of the clones' expression and the pathologists' evaluations were analyzed (Figure 5). All of the ICC values were greater than $0.9(0.939,0.977$ and 0.977$)$. At the $1 \%$ cutoff, Cohen's kappa value for both antibody clones $(22 \mathrm{C} 3$ and $\mathrm{E} 1 \mathrm{~L} 3 \mathrm{~N})$ was 0.893 . The percentages for the OPA, PPA, and NPA were $94.7 \%, 89.9 \%$, and $98.9 \%$, respectively. At the $50 \%$ cutoff, the Cohen's kappa value was 0.868 , and the percentages for the OPA, PPA and NPA were $96.5 \%, 85.7 \%$, and $98.9 \%$, respectively.

For pathologists's evaluation of clone 22C3 expression at the $1 \%$ and $50 \%$ cutoffs, the Cohen's kappa values were 0.941 and 0.912 , respectively, and for the clone E1L3N assay, the Cohen's kappa values were 0.904 and 0.909 at the $1 \%$ and $50 \%$ cutoffs, respectively (Table 3 ).

\section{Discussion}

Presently, the PD-L1 22C3 PharmDx assay, using the Dako Autostainer Link-48 platform, is approved by the Food and Drug Administration (FDA) as a diagnostic companion with pembrolizumab treatment. Though, since the cost of the PD-L1 22C3 PharmDx assay is prohibitive for most laboratories, more cost-effective alternatives are desirable. Considering that standardized assays are expensive and 

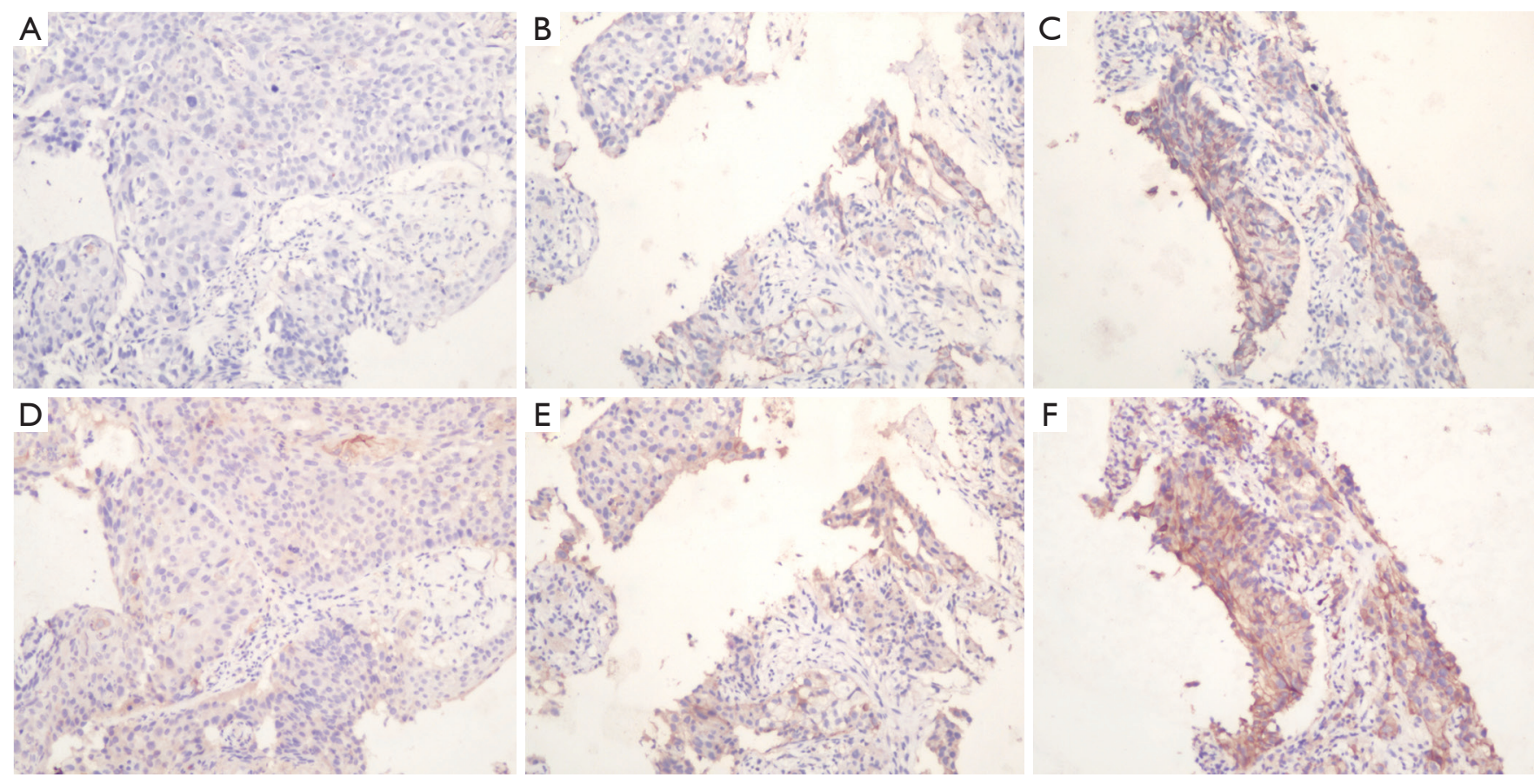

Figure 1 Representative PD-L1 TPS for two clones at three cutoffs (immunohistochemistry, $\times 10$ ). For 22C3, (A) PD-L1 with no staining (score: 0\%); (B) PD-L1 score in the range of 1-49\%; (C) PD-L1 score $250 \%$. For E1L3N, (D) PD-L1 negative (score: 0\%); (E) PD-L1 score in the range from $1-49 \%$; (F) PD-L1 score $\geq 50 \%$.
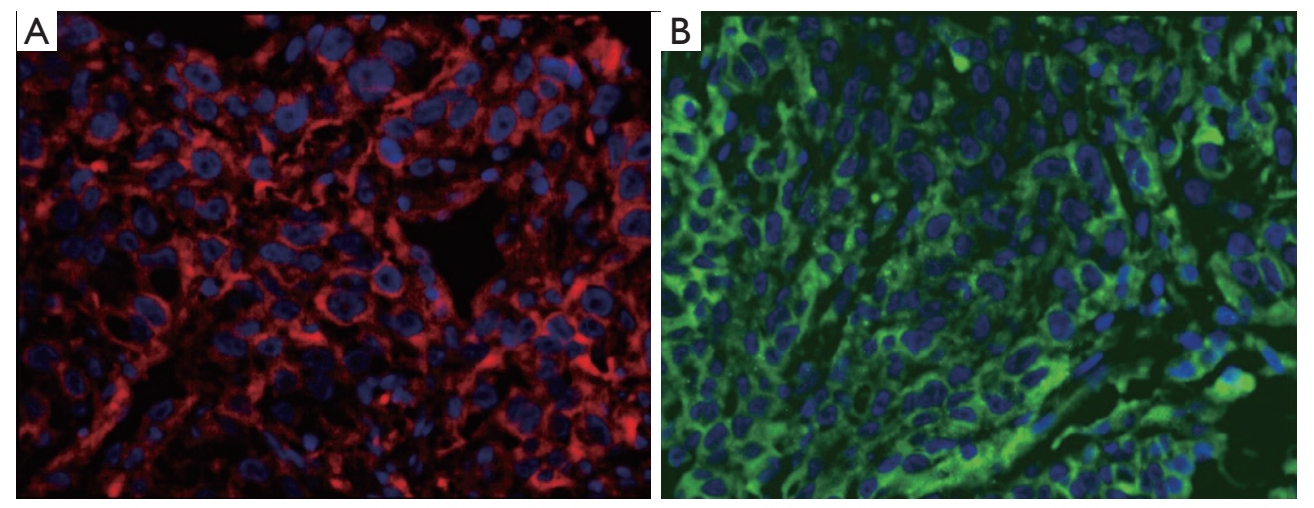

Figure 2 Immunofluorescence staining of PD-L1 antibodies on biopsy samples (40x). (A) Red signal for clone 22C3; (B) green signal for clone E1L3N.

that not all platforms are available in most laboratories, pathologists strongly advocate for a comprehensive evaluation of the diagnostic performance of LDTs (28).

In this regard, harmonization studies that analyze the results obtained from different antibodies have been conducted. Since the clone 22C3 LDT achieved a high concordance with the $22 \mathrm{C} 3$ pharmDx assay on a dedicated Dako Autostainer Link-48 platform (18), the concordance of two LDTs expressions (between the clone $22 \mathrm{C} 3$ concentrated antibody and the clone E1L $3 \mathrm{~N}$ ) is a comparable and meaningful way to analyze the results.

Several studies, such as the Blueprint Projects $(8,9)$, have compared the PD-L1 antibody expression using different clones and testing platforms. Three other PD-L1 clones (28-8, SP263, and SP142) differ in terms of their IHC platform and detection systems. Different PD-L1 IHC 
A

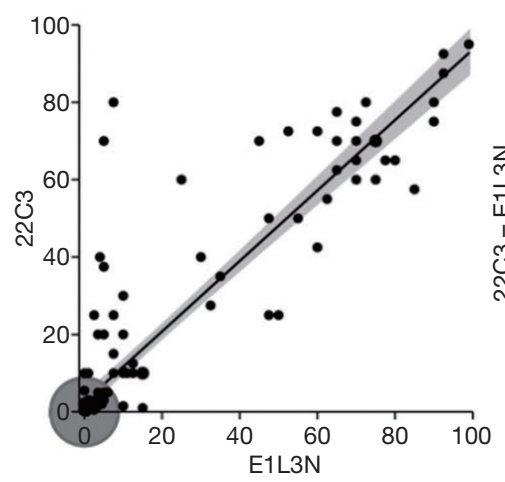

$95 \% \mathrm{Cl} \longrightarrow$ Fitted values
B

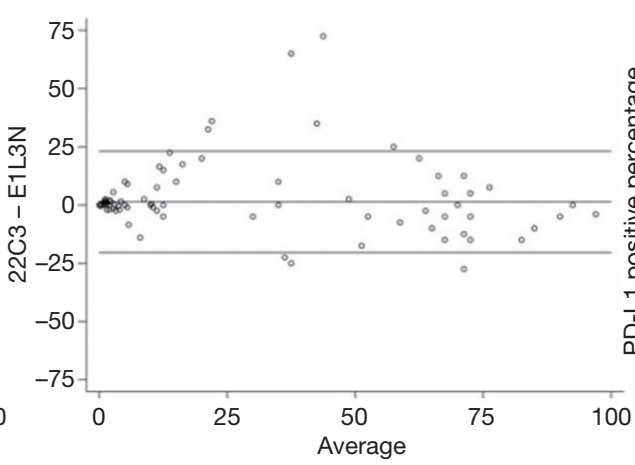

C

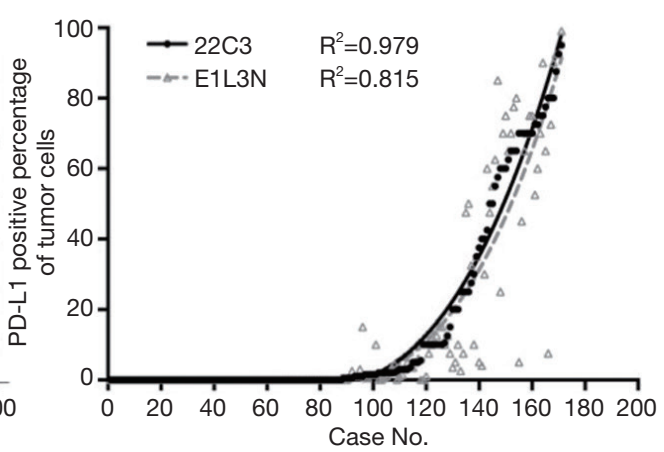

Figure 3 Programmed death-ligand 1 (PD-L1) scoring distribution and comparison of the two clones expression. (A) PD-L1 TPS distribution (22C3 vs. E1L3N) by scatter plot; (B) the difference value (22C3 vs. E1L3N) by Bland-Altman plot; (C) Nonliar fit curve for two clones $(22 \mathrm{C} 3 v s . \mathrm{E} 1 \mathrm{~L} 3 \mathrm{~N})$.

A
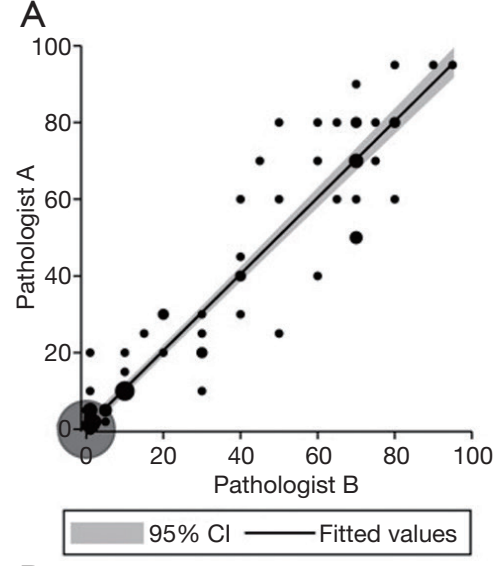

D

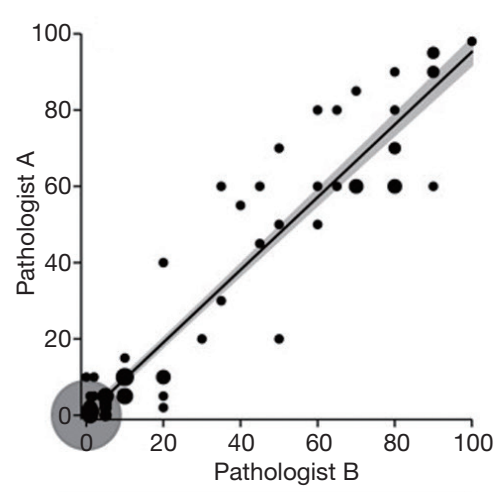

B

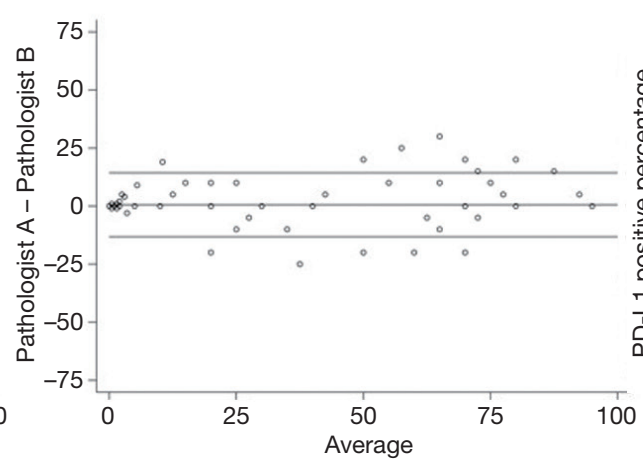

$\mathrm{E}$
C

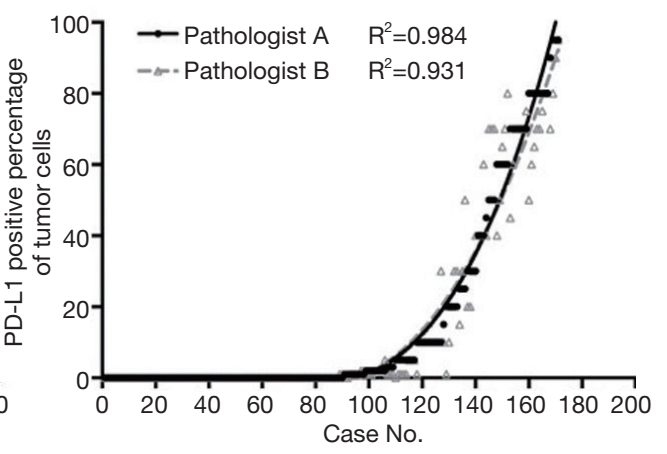

$\mathrm{F}$

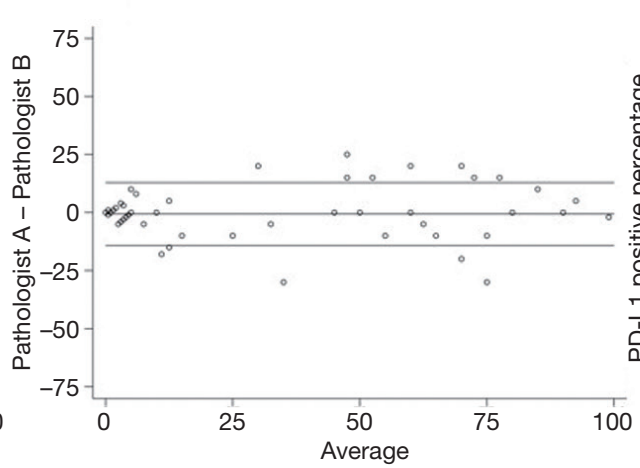

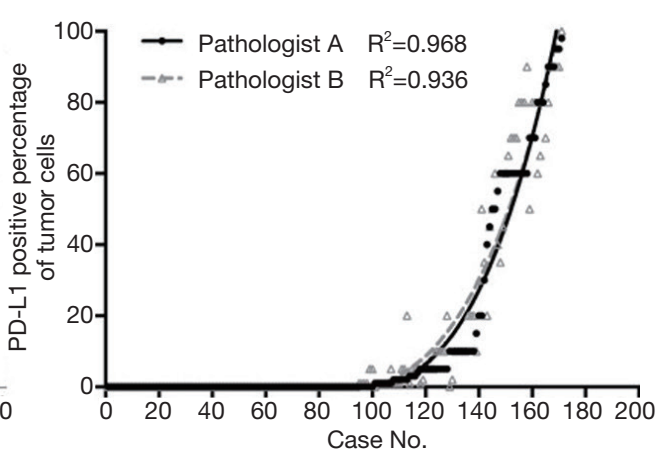

$95 \% \mathrm{Cl}$-Fitted values

Figure 4 Programmed death-ligand 1 (PD-L1) scoring and the concordance between the two pathologists. (A) Scatter plot (Pathologist A vs. Pathologist B) for 22C3; (B) Bland-Altman plot (Pathologist A vs. Pathologist B) for 22C3; (C) Nonliar fit curve of the two pathologists (Pathologist A vs. Pathologist B) for 22C3; (D) Scatter plot of the two pathologists (Pathologist A vs. Pathologist B) for E1L3N; (E) BlandAltman plot of the two pathologists (Pathologist A vs. Pathologist B) for E1L3N; (F) Nonliar fit curve of the two pathologists (Pathologist A vs. Pathologist B) for E1L3N. 
Table 2 Positive percentage of PD-L1 for two clones based on two cutoffs value

\begin{tabular}{lccccccc}
\hline \multirow{2}{*}{ Assay } & \multicolumn{3}{c}{ Cutoff $1 \%, \mathrm{n}(\%)$} & & \multicolumn{3}{c}{ Cutoff 50\%, $\mathrm{n}(\%)$} \\
\cline { 2 - 4 } \cline { 6 - 8 } & Mean & Pathologist A & Pathologist B & & Mean & Pathologist A & Pathologist B \\
\hline E1L3N & $79(46.2)$ & $81(47.4)$ & $80(46.8)$ & & $28(16.4)$ & $27(15.8)$ & $27(15.8)$ \\
\hline
\end{tabular}

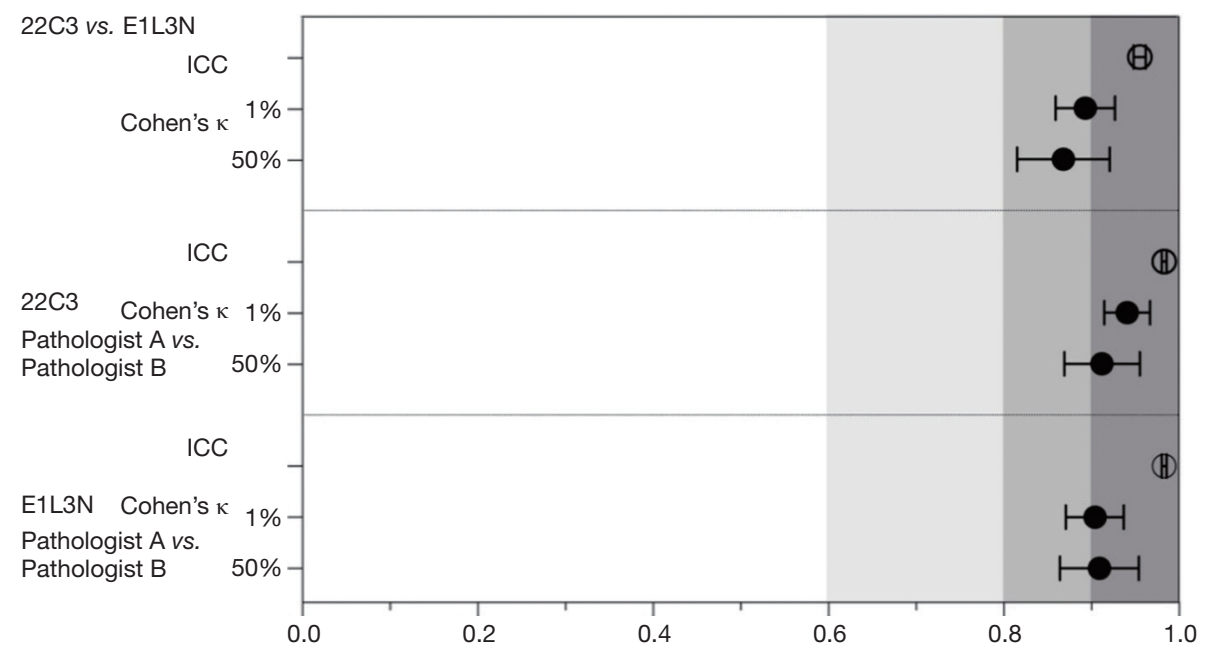

Figure 5 Forest plot for the concordance of the two clones and two pathologists.

Table 3 The concordance of evaluating the two PD-L1 antibody clones between pathologists

\begin{tabular}{|c|c|c|c|c|c|c|}
\hline PD-L1 & $P$ & Cutoff & Cohen's $\kappa$ & OPA (\%) & PPA (\%) & NPA (\%) \\
\hline Pathologist $A$ & & $1 \%$ & 0.893 & 94.7 & 89.9 & 98.9 \\
\hline Pathologist B & & $50 \%$ & 0.868 & 96.5 & 85.7 & 98.6 \\
\hline $22 \mathrm{C} 3$ & 0.154 & & & & & \\
\hline Pathologist B & & $50 \%$ & 0.912 & 97.7 & 92.6 & 98.6 \\
\hline E1L3N & 0.120 & & & & & \\
\hline Pathologist $A$ & & $1 \%$ & 0.904 & 95.3 & 92.0 & 94.0 \\
\hline Pathologist B & & $50 \%$ & 0.909 & 97.7 & 88.9 & 97.9 \\
\hline
\end{tabular}

assays have shown conflicting results (16,23). There may be several reasons for these discrepancies, including the expression heterogeneity in tumors, inter-clone differences, different platforms, and inter/intra-observer variability.

A recent study compared the LDT clone E1L3N to the $22 \mathrm{C} 3$ and SP263 assays to evaluate diagnostic accuracy. Compared to the $22 \mathrm{C} 3$ assay on a dedicated platform, the clone E1L3N showed a lower concordance at $1 \%$ and $50 \%$ cutoffs on the Ventana platform. However, E1L3N was highly consistent with the SP263 assays at both cutoffs using the Ventana platform (16). We found a high concordance of clone E1L3N and 22C3 expression at both the $1 \%$ and $50 \%$ cutoffs on the Dako Autostainer Link-48 platform. Also, clones E1L3N and 22C3 showed a high level of 
concordance by using quantitative immunofluorescence to assess PD-L1 expression. It is, therefore, reasonable to assume that the discrepancies between different PD-L1 IHC assays may be due to the different platforms and detection systems used.

The expression of clones E1L3N, SP142, and 28-8 was compared in surgically resected gastric cancer samples. The findings suggested that E1L3N expressed poor staining in both tumor and immune/stromal cells $(13,29)$. Similarly, quantitative immunofluorescence was applied in resected NSCLC samples to assess the PD-L1 protein. E1L3N showed discordance with clone SP142 (30), which may be attributable to heterogeneity within tumors and antibody affinities. Using an immunohistochemical analysis of PD-L1 protein expression, the antibody E1L3N expressed a slightly higher statistically significant staining than that of $22 \mathrm{C} 3$ based on the average of the pathologists' scores (31). The result demonstrates clone E1L3N is reproducible and that it is interchangeable with clone 22C3 for PD-L1 IHC staining.

Recent studies have evaluated PD-L1 expression between surgically resected samples and paired biopsies, and in these studies, PD-L1 clones exhibited a high level of concordance (32). Additionally, some TMA made from the surgically resected specimen also showed high consistency with the biopsy samples (33-35). These reports indicated that a biopsy is sufficient to represent the entire tumor for detecting the PD-L1 protein.

The high concordance between clone E1L $3 \mathrm{~N}$ and $22 \mathrm{C} 3$ expression was found in this study in three aspects. Firstly, the antibody' design and products' quality control objectively produced reliable assays from both antibody clones. Secondly, the two thoracic pathologists undertook a 22C3 IHC evaluation training course and had experience in cytology and biopsy diagnosis in clinical practice, these results meant that our data was reproducible, and high PPAs were achieved. Lastly, the limited tumor cell area made it easy for the pathologists to obtain an objective TPS compared to the resected NSCLC samples. A previous study found that the inter-pathologist variability was higher than assay variability (36). For this reason, further emphasis should be placed on the training of pathologists to improve their ability to evaluate PD-L1 protein expression (37).

In our study, we systemically compared the PD-L1 antibody E1L3N to the clone 22C3. We found a high concordance between E1L3N and 22C3 expression in NSCLC biopsy samples using IHC on the Dako Autostainer Link-48 platform, indicating that E1L3N could be applied for routine immunohistochemical analysis of PD-L1 detection in NSCLC. Currently, the clone E1L3N is labeled for research use only. Though, due to its costeffectiveness and widespread availability, it is suitable for use in IHC and should be urgently standardized in the operating procedure for LDTs. The results observed in our study demonstrate that clone E1L $3 \mathrm{~N}$ is a promising and interchangeable alternative to the expensive and platformdependent clone $22 \mathrm{C} 3$ assay, especially in research settings. Also, the intra-cellular domain of the E1L3N immunogen was different from $22 \mathrm{C} 3$, whose immunogen was located in the extra-cellular domain containing four glycosylated sites, which might affect the affinity between the PD-L1 epitope and its antibody.

Several limitations should be noted. This study had no outcome data based on of the actual PD-L1 expression status, and the sensitivity and specificity could not be calculated. Also, there was no validation by the commercial PD-L1 IHC 22C3 pharmDx, which is approved by the FDA. However, the concentrated PD-L1 antibody clone $22 \mathrm{C} 3$ achieved an almost equal potency with $22 \mathrm{C} 3$ pharmDx, and it was applied in most of the laboratories with companion instruments. Lastly, the lack of data available for the response to the anti-PD-1/anti-PD-L1 inhibitor therapy in these NSCLC patients presents a further limitation of this study.

\section{Conclusions}

In conclusion, the 22C 3 and E1L3N PD-L1 antibody clones had a high concordance in the PD-L1 IHC staining in NSCLC biopsy samples, even though they were derived from different fragments of PD-L1 peptides and were sourced from different companies. Our results indicate that a more cost-effective LDT with E1L3N could be developed for the routine screening of PD-L1 expression status, especially in a research environment.

\section{Acknowledgments}

Funding: This study was partly supported by projects of the Science and Technology Commission of Shanghai Municipality (No. 18411962900), National Natural Science Foundation of China (No. 81702243 and No. 81802803) and Shanghai municipal health commission (No. 201740134 and No. 20184Y0222) and General program of Xinjiang Natural Science Foundation (2018D01C027). 


\section{Footnote}

Reporting Checklist: The authors have completed the STROBE reporting checklist. Available at http://dx.doi. org/10.21037/tcr-20-101

Data Sharing Statement: Available at http://dx.doi. org/10.21037/tcr-20-101

Conflicts of Interest: All authors have completed the ICMJE uniform disclosure form (available at http://dx.doi. org/10.21037/tcr-20-101). The authors have no conflicts of interest to declare.

Ethical Statement: The authors are accountable for all aspects of the work in ensuring that questions related to the accuracy or integrity of any part of the work are appropriately investigated and resolved. The study was conducted in accordance with the Declaration of Helsinki (as revised in 2013), and approved by the Ethics Committee of Shanghai Pulmonary Hospital (No. k17-130, Shanghai, China). Informed consent was taken from all the patients.

Open Access Statement: This is an Open Access article distributed in accordance with the Creative Commons Attribution-NonCommercial-NoDerivs 4.0 International License (CC BY-NC-ND 4.0), which permits the noncommercial replication and distribution of the article with the strict proviso that no changes or edits are made and the original work is properly cited (including links to both the formal publication through the relevant DOI and the license). See: https://creativecommons.org/licenses/by-nc-nd/4.0/.

\section{References}

1. Siegel RL, Miller KD, Jemal A. Cancer statistics, 2018. CA Cancer J Clin 2018;68:7-30.

2. Bray F, Ferlay J, Soerjomataram I, et al. Global cancer statistics 2018: GLOBOCAN estimates of incidence and mortality worldwide for 36 cancers in 185 countries. CA Cancer J Clin 2018;68:394-424.

3. Horn L, Spigel DR, Vokes EE, et al. Nivolumab Versus Docetaxel in Previously Treated Patients With Advanced Non-Small-Cell Lung Cancer: Two-Year Outcomes From Two Randomized, Open-Label, Phase III Trials (CheckMate 017 and CheckMate 057). J Clin Oncol 2017;35:3924-33.

4. Brahmer JR, Tykodi SS, Chow LQ, et al. Safety and activity of anti-PD-L1 antibody in patients with advanced cancer. N Engl J Med 2012;366:2455-65.

5. Topalian SL, Hodi FS, Brahmer JR, et al. Safety, activity, and immune correlates of anti-PD-1 antibody in cancer. N Engl J Med 2012;366:2443-54.

6. Mok TSK, Wu YL, Kudaba I, et al. Pembrolizumab versus chemotherapy for previously untreated, PD-L1expressing, locally advanced or metastatic non-small-cell lung cancer (KEYNOTE-042): a randomised, open-label, controlled, phase 3 trial. Lancet 2019;393:1819-30.

7. Ettinger DS, Wood DE, Aisner DL, et al. Non-Small Cell Lung Cancer, Version 5.2017 Clinical Practice Guidelines in Oncology. Journal of the National Comprehensive Cancer Network 2017;15:504-35.

8. Hirsch FR, McElhinny A, Stanforth D, et al. PDL1 Immunohistochemistry Assays for Lung Cancer: Results from Phase 1 of the Blueprint PD-L1 IHC Assay Comparison Project. J Thorac Oncol 2017;12:208-22.

9. Tsao MS, Kerr KM, Kockx M, et al. PD-L1 Immunohistochemistry Comparability Study in Real-Life Clinical Samples: Results of Blueprint Phase 2 Project. J Thorac Oncol 2018;13:1302-11.

10. Batenchuk C, Albitar M, Zerba K, et al. A real-world, comparative study of FDA-approved diagnostic assays PD-L1 IHC 28-8 and 22C3 in lung cancer and other malignancies. J Clin Pathol 2018;71:1078-83.

11. Yun S, Park Y, Moon S, et al. Clinicopathological and prognostic significance of programmed death ligand 1 expression in Korean melanoma patients. J Cancer 2019;10:3070-8.

12. Tretiakova M, Fulton R, Kocherginsky M, et al. Concordance study of PD-L1 expression in primary and metastatic bladder carcinomas: comparison of four commonly used antibodies and RNA expression. Mod Pathol 2018;31:623-32.

13. Ma J, Li J, Qian M, et al. PD-L1 expression and the prognostic significance in gastric cancer: a retrospective comparison of three PD-L1 antibody clones (SP142, 28-8 and E1L3N). Diagn Pathol 2018;13:91.

14. Mo RJ, Han ZD, Liang YK, et al. Expression of PD-L1 in tumor-associated nerves correlates with reduced CD8(+) tumor-associated lymphocytes and poor prognosis in prostate cancer. Int J Cancer 2019;144:3099-110.

15. Downes MR, Slodkowska E, Katabi N, et al. Inter- and intraobserver agreement of programmed death ligand 1 scoring in head and neck squamous cell carcinoma, urothelial carcinoma and breast carcinoma. Histopathology 2020;76:191-200.

16. Munari E, Zamboni G, Lunardi G, et al. PD-L1 
expression in non-small cell lung cancer: evaluation of the diagnostic accuracy of a laboratory-developed test using clone E1L3N in comparison with 22C3 and SP263 assays. Hum Pathol 2019;90:54-9.

17. Martinez-Morilla S, McGuire J, Gaule P, et al. Quantitative assessment of PD-L1 as an analyte in immunohistochemistry diagnostic assays using a standardized cell line tissue microarray. Lab Invest 2020;100:4-15.

18. Adam J, Le Stang N, Rouquette I, et al. Multicenter harmonization study for PD-L1 IHC testing in non-smallcell lung cancer. Ann Oncol 2018;29:953-8.

19. Marchetti A, Barberis M, Franco R, et al. Multicenter Comparison of 22C3 PharmDx (Agilent) and SP263 (Ventana) Assays to Test PD-L1 Expression for NSCLC Patients to Be Treated with Immune Checkpoint Inhibitors. J Thorac Oncol 2017;12:1654-63.

20. Sughayer MA, Alnaimy F, Alsughayer AM, et al. Comparison of 22C3 PharmDx and SP263 Assays to Test PD-L1 Expression in NSCLC. Appl Immunohistochem Mol Morphol 2019;27:663-6.

21. Ratcliffe MJ, Sharpe A, Midha A, et al. Agreement between Programmed Cell Death Ligand-1 Diagnostic Assays across Multiple Protein Expression Cutoffs in Non-Small Cell Lung Cancer. Clin Cancer Res 2017;23:3585-91.

22. Munari E, Rossi G, Zamboni G, et al. PD-L1 Assays 22C3 and SP263 are Not Interchangeable in Non-Small Cell Lung Cancer When Considering Clinically Relevant Cutoffs: An Interclone Evaluation by Differently Trained Pathologists. Am J Surg Pathol 2018;42:1384-9.

23. Hendry S, Byrne DJ, Wright GM, et al. Comparison of Four PD-L1 Immunohistochemical Assays in Lung Cancer. J Thorac Oncol 2018;13:367-76.

24. Lee KS, Choe G, Yun S, et al. Comparative analysis of programmed cell death ligand 1 assays in renal cell carcinoma. Histopathology 2020;77:67-78.

25. Agilent. PD-L1 IHC 22C3 pharmDx Interpretation Manual. 2016. Available online: https:/www.agilent.com/ cs/library/usermanuals/public/29158_pd-11-ihc-22C3pharmdx-nsclc-interpretation-manual.pdf

26. Roach C, Zhang N, Corigliano E, et al. Development of a Companion Diagnostic PD-L1 Immunohistochemistry Assay for Pembrolizumab Therapy in Non-Small-cell Lung Cancer. Appl Immunohistochem Mol Morphol 2016;24:392-7.

27. Dolled-Filhart M, Roach C, Toland G, et al. Development of a Companion Diagnostic for Pembrolizumab in NonSmall Cell Lung Cancer Using Immunohistochemistry for Programmed Death Ligand-1. Arch Pathol Lab Med 2016;140:1243-9.

28. Büttner R, Gosney JR, Skov BG, et al. Programmed Death-Ligand 1 Immunohistochemistry Testing: A Review of Analytical Assays and Clinical Implementation in NonSmall-Cell Lung Cancer. J Clin Oncol 2017;35:3867-76.

29. Gaule P, Smithy JW, Toki M, et al. A Quantitative Comparison of Antibodies to Programmed Cell Death 1 Ligand 1. JAMA Oncol 2017;3:256-9.

30. McLaughlin J, Han G, Schalper KA, et al. Quantitative Assessment of the Heterogeneity of PD-L1 Expression in Non-Small-Cell Lung Cancer. JAMA Oncol 2016;2:46-54.

31. Rimm DL, Han G, Taube JM, et al. A Prospective, Multi-institutional, Pathologist-Based Assessment of 4 Immunohistochemistry Assays for PD-L1 Expression in Non-Small Cell Lung Cancer. JAMA Oncol 2017;3:1051-8.

32. Kitazono S, Fujiwara Y, Tsuta K, et al. Reliability of Small Biopsy Samples Compared With Resected Specimens for the Determination of Programmed Death-Ligand 1 Expression in Non--Small-Cell Lung Cancer. Clin Lung Cancer 2015;16:385-90.

33. Gniadek TJ, Li QK, Tully E, et al. Heterogeneous expression of PD-L1 in pulmonary squamous cell carcinoma and adenocarcinoma: implications for assessment by small biopsy. Mod Pathol 2017;30:530-8.

34. Munari E, Zamboni G, Marconi M, et al. PD-L1 expression heterogeneity in non-small cell lung cancer: evaluation of small biopsies reliability. Oncotarget 2017;8:90123-31.

35. Munari E, Zamboni G, Lunardi G, et al. PD-L1 Expression Heterogeneity in Non-Small Cell Lung Cancer: Defining Criteria for Harmonization between Biopsy Specimens and Whole Sections. J Thorac Oncol 2018;13:1113-20.

36. Brunnström H, Johansson A, Westbom-Fremer S, et al. PD-L1 immunohistochemistry in clinical diagnostics of lung cancer: inter-pathologist variability is higher than assay variability. Mod Pathol 2017;30:1411-21.

37. Savic Prince S, Bubendorf L. Predictive potential and need for standardization of PD-L1 immunohistochemistry. Virchows Arch 2019;474:475-84.

Cite this article as: Zhang W, Cao Z, Gao C, Huang Y, Wu C, Zhang L, Hou L. High concordance of programmed death-ligand 1 expression with immunohistochemistry detection between antibody clones $22 \mathrm{C} 3$ and E1L3N in nonsmall cell lung cancer biopsy samples. Transl Cancer Res 2020;9(10):5819-5828. doi: 10.21037/tcr-20-101 
Supplementary

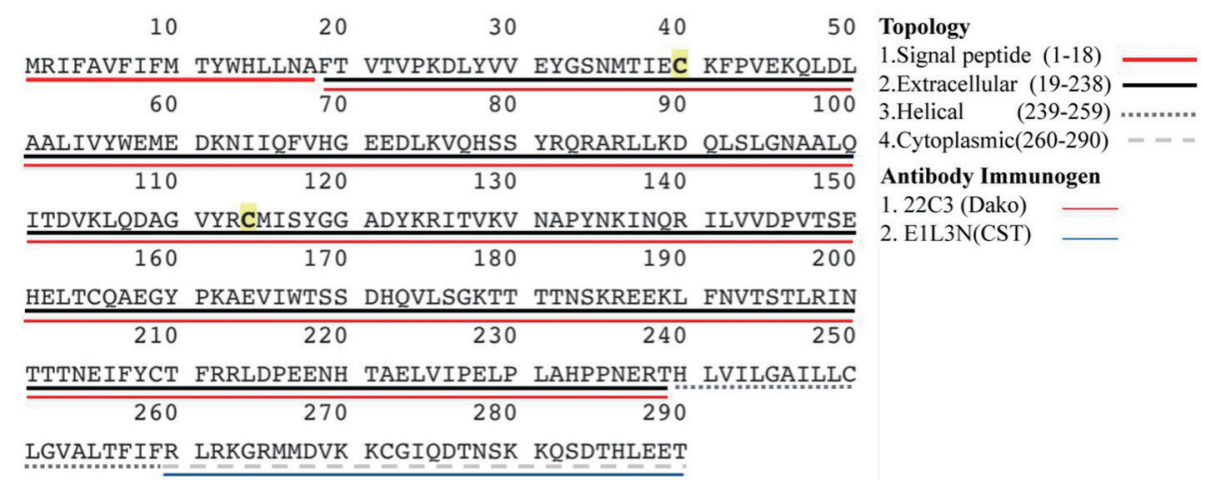

Figure S1 Programmed death-ligand 1 (PD-L1) topology and the immunogen of both antibody clone E1L3N and 22C3. 